\title{
Review \\ Review of an Explanation of Aerodynamic Lift by Triple Deck Theory
}

\author{
Alois Peter Schaffarczyk $1,+, \ddagger$ (iD \\ 1 Kiel University of Applied Sciences, Grenzstrasse 3, D-24149 Kiel, Germany; Alois.Schaffarczyk@FH-Kiel.de \\ * Correspondence: Alois.Schaffarczyk@FH-Kiel.de; Tel.: +49-431-210-2610
}

\begin{abstract}
Inspired from a recent article by Regis [1], earlier publised work of McLean [2-4] and informal discussions much earlier with members of the Danish Technical University and KTH, Sweden we summarize known results for viscous correction to the lift coefficient for thin aerodynamic profiles. We thereby try to remember the work of [5] on triple-deck or higher order Boundary Layer theory and compare it to simple 2D Computational Fluid Dynamic (CFD) models. As a conclusion, a clearer definition of terms like understanding and explanation applied to the phenomena of aerodynamic lift will be given.
\end{abstract}

Keywords: Aerodynamic Lift, Boundary Layer Theory, Triple Deck Theory

\section{Introduction: What is an explanation?}

People often ask: I do not understand! Can you explain it again? Then a lecturer has to do his (or her) best to find ways, such that, the person asking the question gets a better feeling of understanding. Some people even say: You have only understood something if you can explain it to other people. But this only shifts the problem to these other people.

Even more complicated and since the emergence of Quantum Physics, some say that an understanding of certain phenomena is simply impossible. Fortunately, Fluid Mechanics is what is termed Classical Mechanics and may be related to every-day experiences and is thus much easier to explain than quantum mechanical phenomena.

However, sometimes it seems that a discussion around aerodynamic lift is closer to quantum mechanics then to classical mechanics which may be related to the fact, that the mathematical description is classical but in terms of a non-linear field-theory.

Here we take the following point of view:

1. We have a theory (or model) for some phenomena if we have a set of assumptions resulting in equations for quantitative descriptions to be compared with measurements.

2. Pure numerical solutions from the most basic equations are not sufficient as they only produce very specific results.

We shortly refer to Newton's $2^{\text {nd }}$ law for a point mass:

$$
F=\dot{p}
$$

A force (in $\mathrm{N}$ ) relates to the temporal change of momentum $p=m \cdot v$. A cause and effect relationship may be established in both directions, meaning that a force is a cause for a change in momentum, or a change in momentum may be the cause for an inertial force.

Fluid mechanics as a continuum theory is formulated in terms of a velocity field $\mathbf{v}$ and expresses momentum change (mChange) and mass conservation (mCons):

$$
\begin{aligned}
\text { mChange: } \rho\left(\frac{\partial \mathbf{v}}{\partial t}+(\mathbf{v} \cdot \nabla) \mathbf{v}\right) & & =\mathbf{f}-\nabla p+\mu \Delta \mathbf{v}, \\
\text { mCons: } \nabla \cdot \mathbf{v} & & =0 .
\end{aligned}
$$


Instead of the quantity force it introduces a static pressure $p$ and a volume force density $\left(\mathrm{N} / \mathrm{m}^{3}\right)$. To calculate a force on an extended body (airfoils) one has to integrate pressure (and viscous shear stress) on its surface. By use of Eq. (3) pressure can be eliminated but the description is non-local:

$$
\Delta p=-\rho \frac{\partial^{2} u_{i} u_{j}}{\partial x_{i} \partial x_{j}}:=S(\mathbf{r})
$$

Formally this equation may then be solved by the introduction of Green's function:

$$
p(\mathbf{r})=p^{\text {harmonic }}(\mathbf{r})+p(\mathbf{r})+\frac{\rho}{4 \pi} \int_{\mathbb{R}^{3}} \frac{S(\mathbf{r})}{\left|\mathbf{r}-\mathbf{r}^{\prime}\right|} d \mathbf{r}^{\prime},
$$

where $p^{\text {harmonic }}(\mathbf{r})$ is a solution of the homogeneous pressure equation:

$$
\Delta p^{\text {harmonic }}(\mathbf{r})=0 \text {. }
$$

f usually is regarded as given from outside and in many cases does not have to be included. (With an exception of the so-called Actuator Disk see [6].)

In the rest of the paper we proceed as follows: We review basic models of lift in a more logical manner than they appeared historically, compare them with numerical simulations and conclude with a physical model which in our understanding provides a (long known) explanation of aerodynamic lift.

We have to remark that our review mainly follows an approach in the spirit of Landau [7] which from the beginning emphasizes the role of a wake emerging down stream of an aerodynamic profile. Therefore it is rather different from that of McLean [2-4] who emphasizes the non-local pressure field as a direct result of the lift force [4] and its reciprocal interaction with the velocity field as a key ingredient of a qualitative explanation of lift.

\section{Thin Airfoil Theory}

\subsection{A First Encounter with History of Explanations of Aerodynamic Lift}

Specific shaped 2D sections exhibit a large force perpendicular to the inflow direction. This force is called lift. Nowadays, not only airplanes use it but also most of the highly efficient machines, e.g. helicopters, ship propellers and wind turbines. Nevertheless, even today, there is a discussion [2] for an explanation (in the sense of a cause and effect relation mentioned above) as it was in the beginning of the 20th century. Bloor [8] gives an excellent and very readable review of some of the early historical developments from about 1900 to 1930. In short two schools used either Newton's corpuscular picture or the newly emerged circulation model.

\subsection{Inviscid Thin Airfoil theory}

A first important step in developing a theory of aerodynamic lift, of what one may term even an understanding was the formulation of the so-called Kutta-Joukovsky theorem [9] which states

$$
L=-\rho \cdot w \cdot \Gamma .
$$

Here $\mathrm{L}$ is the lift force (per unit span), $\rho$ the density of the fluid, $\mathrm{w}$ the inflow velocity (far up-stream) and $\Gamma$ the circulation, defined as

$$
\Gamma:=\oint_{C} v \cdot d r
$$

$\mathrm{C}$ is an arbitrary closed loop around the airfoil.

Therefore, a dynamical quantity (lift) is connected to fully kinematic quantities $(\mathrm{w}, \Gamma)$, only. Sign conventions are as follows:

1. velocity: left to right,

2. lift: from bottom to top,

3. circulation: counter-clockwise. 


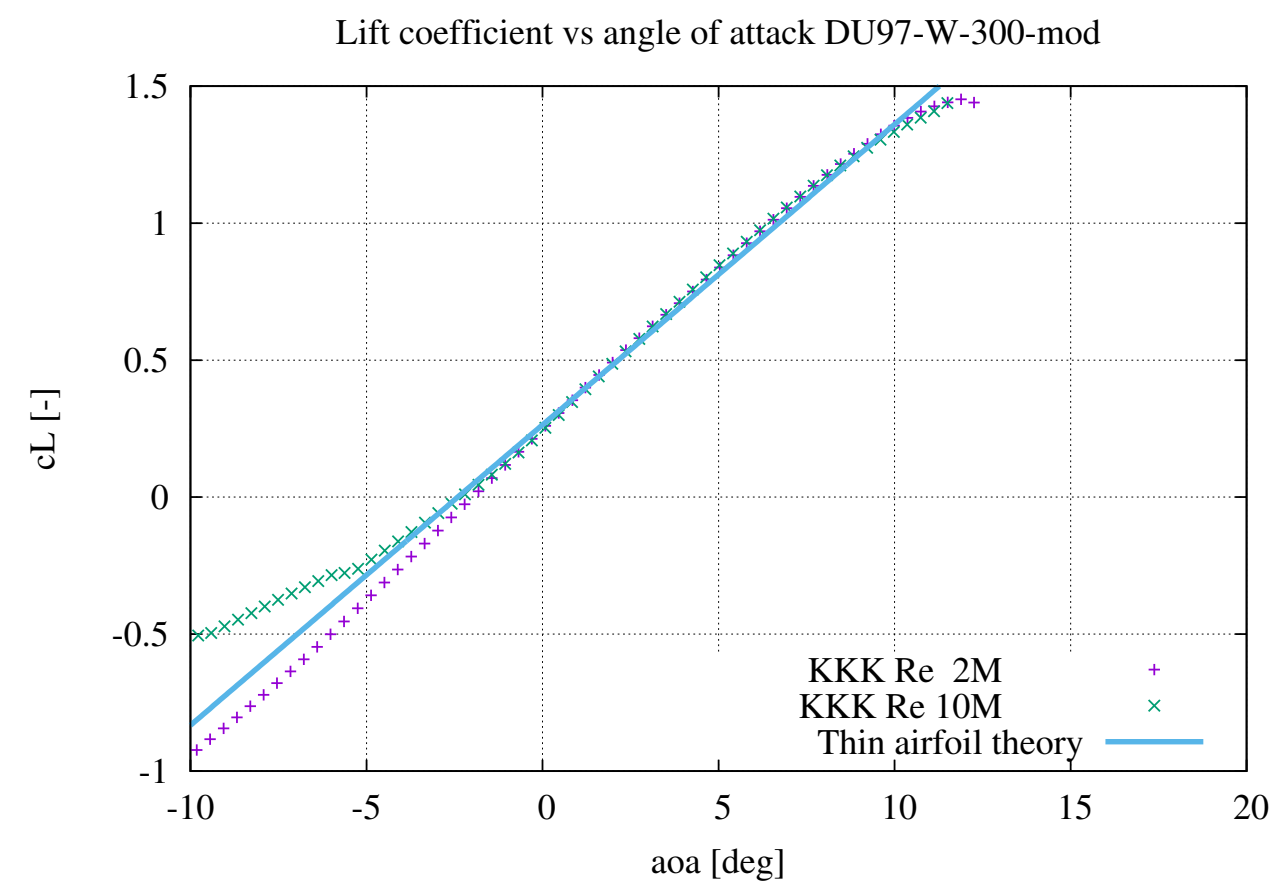

Figure 1. Comparison of measured lift coefficient vs angle-of-attack with thin airfoil theory showing a large region of linear variation close to $c_{L}^{\max }$. Results for RN 2 and 10 million are shown. For RN of $10 \mathrm{M}$ separation on the lower (pressure) side occurs much earlier than for RN of $2 \mathrm{M}$.

This explains the negative sign in Eq. 7.

A unique circulation, needed for a unique defined lift, needs a formulation of additional assumptions and this resulted in the so-called Kutta-condition. It may be stated in various forms. If the airfoil tail $(x / c=1)$ is regarded to have a non-smooth change in geometrical slope from the upper to the lower side (known as the trailing edge), it is usually expressed - as a more mathematical statement - demanding that all velocities should be finite, i.e. $<\infty$, esp. in the vicinity of the tail. Note that in-viscid potential theory does not forbid infinite velocities.

For an ellipse this clearly is not the case, therefore a severe logical loop-hole exists at least for these kind of trailing edges. However, Howarth as early as 1935 [10] managed to calculate lift and drag manually for this particular shape, an ellipse, by what is now called interactive boundary layer theory and its expression is found in the well-known code XFOIL [11]. Sears [12] took these ideas further and formulated corresponding conditions for static pressure at the upper and lower edges of the boundary layers in the sense of generalizing the Kutta-Joukovsky condition to viscous (boundary layer) flow.

The complete transient procedure on how circulation is generated from rest is still under investigation, see for example [13] for a recent CFD work.

Thin airfoil theory (TAT, called that way because any influence of the finite thickness of an airfoil is neglected) [14] gives a remarkable simple expression for the lift-coefficient

$$
c_{l}=2 \pi \cdot\left(\alpha+\alpha_{0}\right) .
$$

A lift-curve slope of $2 \pi$ - independent of all geometrical details - therefore is predicted and the angle-of-attack (AOA) appears to be the most important quantity. Nevertheless, one particular geometrical quantity, camber (f), enters Eq 9 via $\alpha_{0}=2 f$ shifting zero-lift AOA to negative angles. If flow direction is counted positive as when coming from the left, a positive AOA is given when the airfoil is rotated in clock-wise direction.

Fig 1 compares measurements [15] of a 30\% thick airfoil - with $2.1 \%$ camber - dedicated for wind turbine blades with prediction of thin-airfoil-theory. A remarkably large (more 
than 20 degrees) range of agreement (within the experimental uncertainty) even for this certainly not-thin airfoil exists.

\subsection{Viscous Thin Airfoil Theory I: $R N<<1$}

TAT is based on inviscid models of fluid flows (only density as a material enters) and as a consequence, e.g. circulation is a conserved quantity, i.e. it can neither be created nor destroyed. Therefore, more sophistcated models (and equations) must be included if the emergence of lift is to be explained. It is well known that the Navier-Stokes Equations provide this basis, adding a second material parameter, viscosity. In a series of journal and technical papers Yates [16] (and independently Bryant and Williams [17] and Shen and Crimi [18]) with the help of a Oseen-type approximation (in fact a linerisation) were able to use these Navier-Stokes equations to

1. derive and thereby explain the Kutta condition and

2. to give asymptotic corrections to the lift-curve slope in terms of inverse Reynolds number.

This is somewhat surprising as Oseen-Flow, see [9], chapter (4.10), is generally assumed to be valid in low-Re (RN $<1$, creeping) flow only, whereas in high-Re flow (RN $\left.>10^{5}\right)$ boundary layer theory [19] should be more appropriate. As a consequence numerical agreement for changes in the lift-slope (with reference to $2 \cdot \pi$ ) were not convincing.

\subsection{Finite thickness}

In this context, to separate between thickness and viscous effects a lot of authors including Abbot and von Doenhoeff [20] tried to improve (inviscid) TAT by investigating the influence of thickness on the lift-curve-slope which typically results in equations like: [20],

$$
\begin{aligned}
c_{L} & =2 \pi(1+\tau), \\
\tau=\frac{\epsilon}{a} & =\frac{4 \sqrt{3}}{9} \cdot \frac{t}{c} .
\end{aligned}
$$

Yates [21,22] combined Reynolds number and thickness corrections to

$$
c_{L}=2 \pi(1+\tau) \cdot\left(1-\frac{4}{\log (64 R N)+\gamma_{E}}\right)
$$

$\gamma_{E}=0.57722$ being Euler's constant which shows a decrease of more than $10 \%$ at $\mathrm{t} / \mathrm{c}=0.3$ and $\mathrm{RN}$ around $10^{5}$ from $\mathrm{RN}$ effects which - at least - is partly compensated by the first (thickness) term. McLean [2], chapter 7.4, pp 313/314 gives further details.

Not included in all these discussions is the influence of the flow-state of the boundary layer, whether it is laminar or turbulent. In our discussions we assume that lift (in the linear part) is not influenced as strongly as drag. It is well known that drag can be much higher when most parts of the boundary layer are turbulent.

Another important phenomena, flow separation, the starting point defined by

$$
c_{f}=\mu \cdot \frac{d v_{t}}{d n} \leq 0,
$$

is closely related to limiting $c_{L}$ to values around 1.5 (of course with remarkable exceptions).

We implicitly assume that the effect of separation can be approximately described by shifting the trailing edge to the points of separation $[10,12]$. This restricts our discussion to small AOA only $\left(-1^{\circ}<\alpha<5^{\circ}\right)$. 


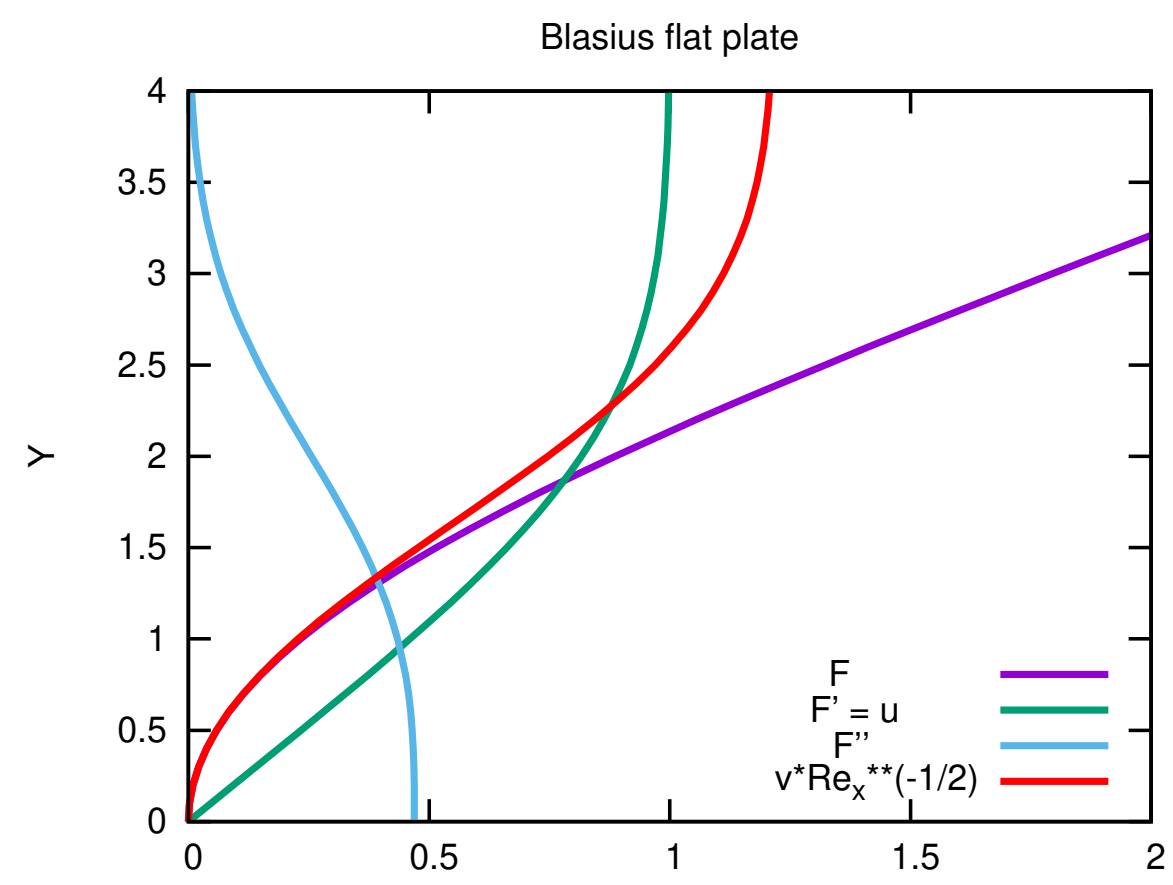

Figure 2. Stream function $(F)$ and velocity profile $\left(F^{\prime}\right)$ from numerical Integration of Eq. $(16) . F^{\prime \prime}(y=0)$ correspond to wall shear stress. In addition, it can be seen that the normal velocity $\mathrm{v}$ approaches a finite value $\sim \sqrt{R N_{x}}$ when the boundary layer edge is reached.

\section{Viscous correction by Schmitz}

Schmitz [23-25] calculated finite domain viscous correction and found small deviations $\left(10^{-2}\right.$ of inviscid circulation) only for an airfoil flow at $\operatorname{Re}=500 \mathrm{k}$ [17]. As a result a typical reduction in $c_{L}$ of

$$
\Delta c_{L}=\sim-2\left(\frac{U_{e}}{U_{\infty}}\right)^{2}\left(\frac{\delta_{T E}}{c}\right)^{2},
$$

is predicted. Here, $U_{e}$ resp. $U_{\infty}$ is the velocity at the edge of the boundary layer at the trailing edge (TE) resp. the inflow velocity; $\delta_{T E}$ is the boundary layer thickness and c the chord of the profile. A simple estimation for $\mathrm{RN} \sim 10^{6}$ shows that the resulting reduction depends on the type of the flow and is comparable small for a pure laminar boundary layer. It is interesting to note that Triple-Deck-Theory (see section 3.5) is able to derive a similar contribution with reads as [?]

$$
\frac{3}{8 \pi c}\left(\delta_{1}+\theta\right) R N^{-1 / 2} \cdot \log (R N) .
$$

Here $\delta_{1}$ and $\theta$ is the displacement thickness and momentum thickness at the trailing edge, resp.

\section{Viscous Thin Airfoil Theory II: $R N>>1$}

\subsection{Boundary Layer Theory}

Boundary layer theory (BLT) started with the seminal paper of Prandtl [26]. As one of the first applications a semi-infinite flat plate located at $x>0$ and $y=0$ was investigated by Blasius [27]. He reduced the Navier-Stokes Equations to a still non-linear but much simpler ordinary differential equation for the stream function F:

$$
F^{\prime \prime \prime}+F \cdot F^{\prime \prime}=0,
$$




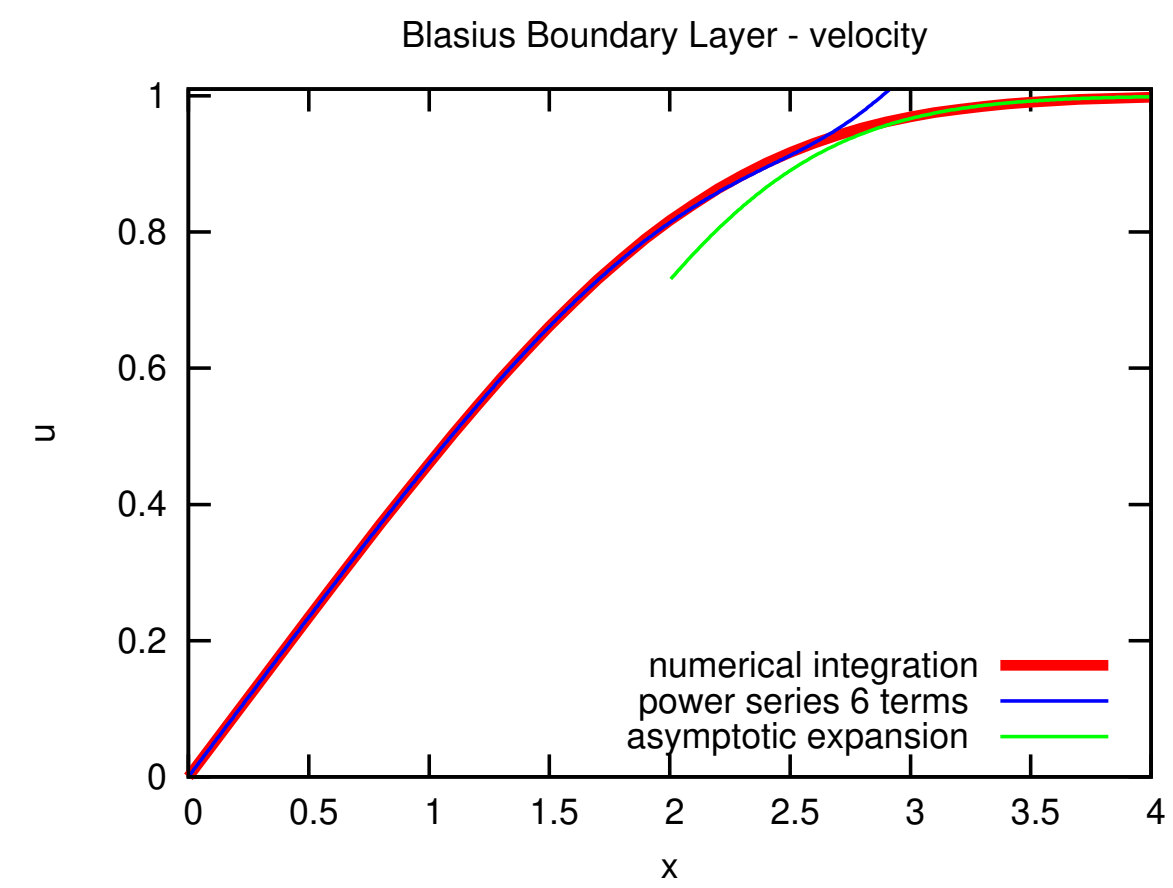

Figure 3. $\mathrm{F}^{\prime}$ (velocity profile) and its representation by a power series and asymptotic expansion. The rather large (more than 14 digits) integer coefficient were calculated with the help of MATHEMATICA (c).

together with boundary conditions $F(0)=F^{\prime}(0)=0$ and $F(s) \rightarrow s$ as $s \rightarrow \infty$. Here $s=Y / \sqrt{2 x}$. Blasius was able to represent the solution as a power series (see [28-30] for mathematical details)

$$
\begin{array}{r}
F(s)=\frac{1}{2} \lambda s^{2}-\frac{1}{240} \lambda^{2} s^{5}+\frac{11}{161280} \lambda^{3} s^{8}+\ldots \\
\text { with: } \lambda=F^{\prime \prime}(y=0) .
\end{array}
$$

With $R e_{x}=u_{\infty} \cdot x / v$ it follows

$$
\begin{gathered}
\delta_{99}=5.0 \cdot R e_{x}^{-1 / 2} \text { at } \mathrm{y} \text { where } u=0.99 \cdot U_{\infty} \\
\delta_{1}=1.72 \cdot R e_{x}^{-1 / 2} \text { displacemenet thickness } .
\end{gathered}
$$

It must be noted, that today Eq. (16) is typically solved numerically to arbitrary accuracy, see Fig 2. The asymptotic behavior $y \rightarrow \infty$ can be studied by assuming

$$
F(y)=y-\beta_{0}+g_{o}(y) .
$$

It follows [31]

$$
\begin{array}{r}
\beta_{0}=1.21649, \\
g_{0}(y)=\exp \left(-\frac{y^{2}}{2}\right) .
\end{array}
$$

Fig. 3 shows the accuracy of both Taylor series around 0 and $\infty$.

\subsection{Drag, Comparison with Experiment and Higher Order Boundary Layer Theory}

As wall shear stress is related to $F^{\prime \prime}(y \rightarrow 0)$ drag can then be calculated by integration and further compared to measurements. Two findings are important:

1. for $R e_{x}>5 \cdot 10^{5}$ flow state starts changing to a turbulent one, 


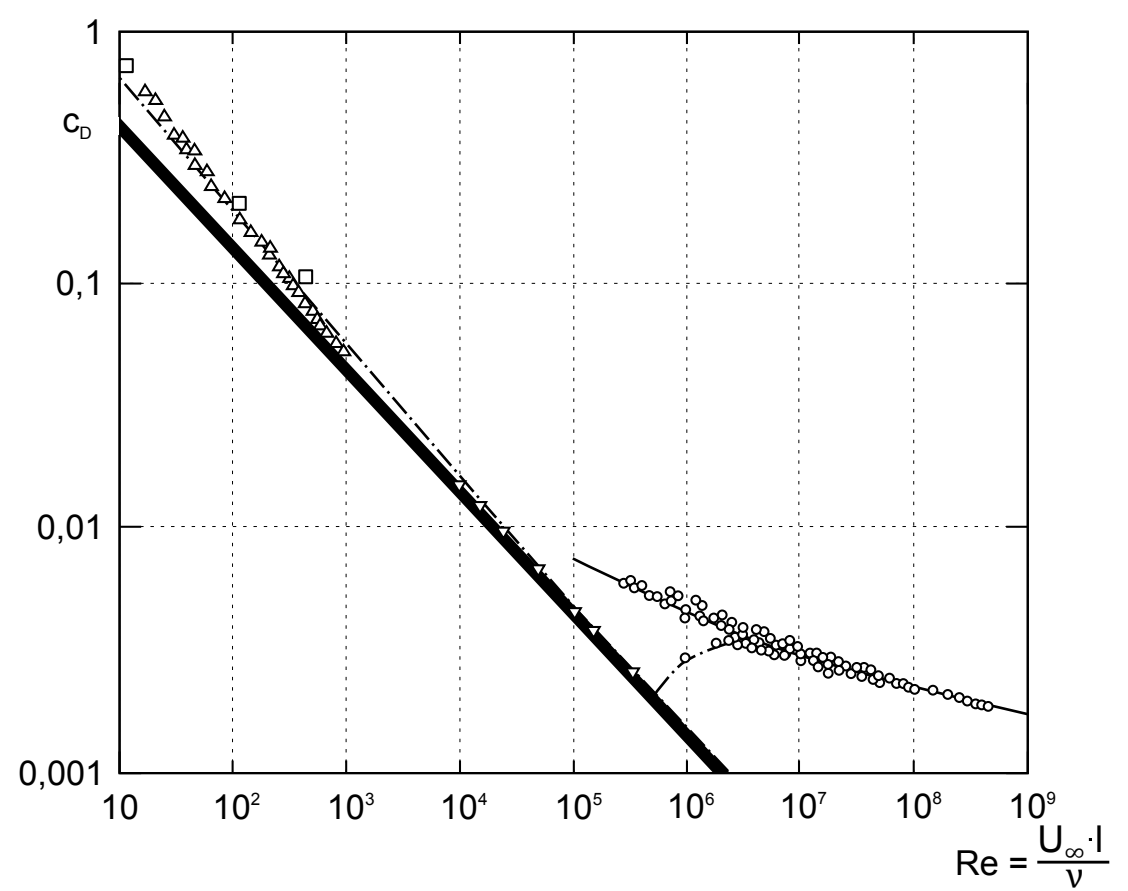

Figure 4. Comparison of drag from BLT and measurements. Fat line: Blasius, dashed line: higher order (Triple Deck) and empirical correlation for the turbulent case

2. for $R e_{x}<10^{4}$ deviations become larger as expected, see Fig 4 .

Improvement is possible if BLT is regarded as an asymptotic expansion in powers of inverse Reynolds number. First order then are terms $\sim R e^{-1 / 2}$. As one can see from the dashed line in Fig 4, there is significant improvement - even down to $R e_{x} \sim 10$ - if one takes higher orders into account as will be seen in section 3.5.

Blasius (solid line):

$c_{D}=2 \cdot 0.664 \cdot(\operatorname{Re})^{-1 / 2}$

2nd order BL (dashed line):

$c_{D}=2 \cdot 0.664 \cdot(R e)^{-1 / 2}+2.67 \cdot(R e)^{-7 / 8}$

values for $\operatorname{Re}=10(1000)$ :

$c_{D}(R e=10(1000))=0.42(0.042)+0.36(0.006)$.

Eq. (25) contains a new term $\sim R e^{-7 / 8}$ which contributes to more than $10 \%$ and which will be derived below.

\subsection{Flat plate of finite length}

Having described the problem of a semi-infinite plate $(x>0)$ we turn to a flat plate of finite length: $-1<x<0$ :

\subsection{Goldstein's inner and outer wake}

Only some years later BLT was extended to a flat plate of finite length $-1<x<$ $0, y=0$ by Goldstein [32]. The situation is as follows: At the plate we have for $\mathrm{y}=0 \mathrm{u}=0$ which is simply the no-slip condition. Within the wake $(x>0)$ we will have $u \neq 0$. This different behavior at $y=0$ for $x<0$ and $x>0$ is the reason, that the wake exhibits a two-fold structure, separated by a curve $y \sim x^{-1 / 3}$, see Fig. 6 . Unfortunately, close to $x=0$ a singularity appears:

$$
v(x, 0) \sim x^{-1 / 3}, x>0,
$$




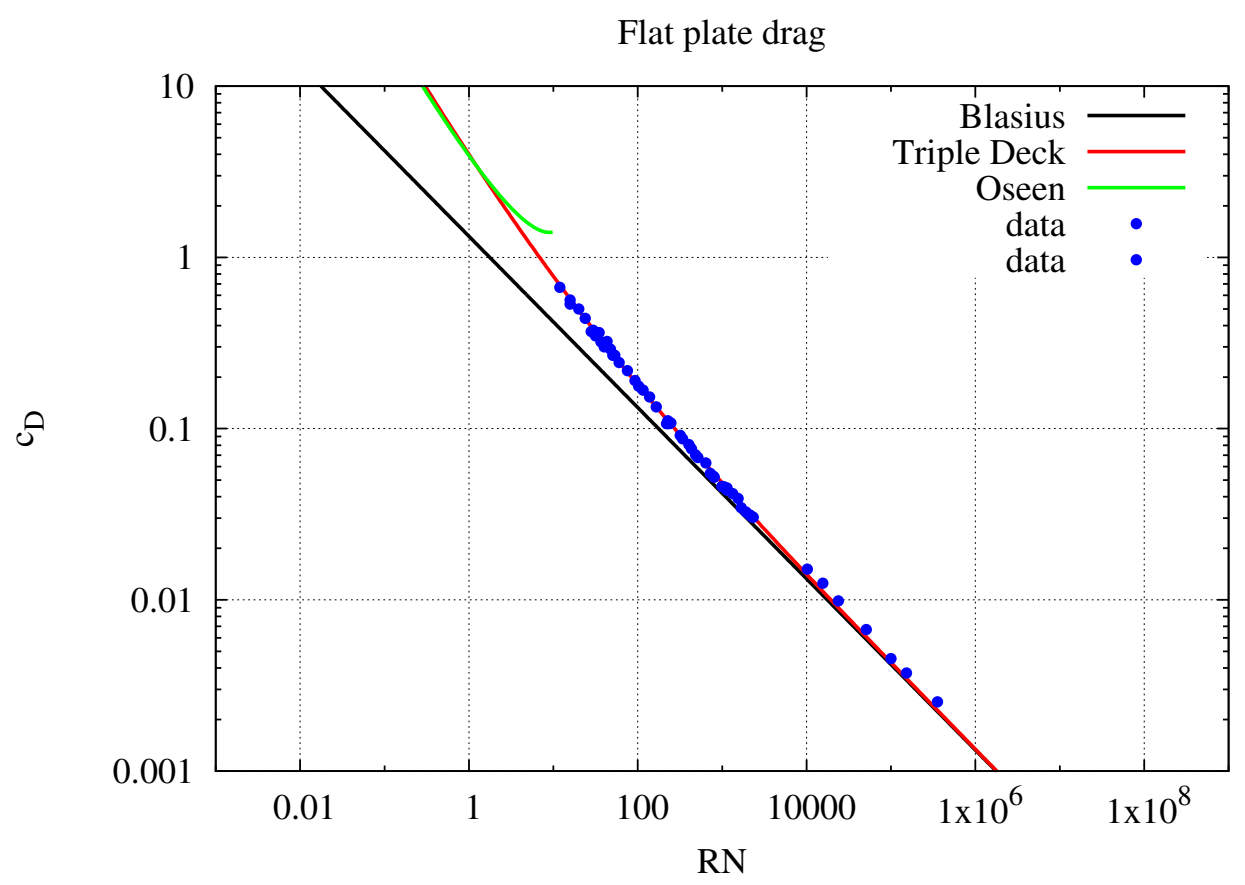

Figure 5. Drag coefficient of a flat plate in the laminar state and from various approaches: Measurements and Theories of Oseen, Blasius and the Triple-Deck-Theory for an extended RN region down to less than RN of $10^{-2}$.

which is named Goldstein singularity and can be calculated from $\Psi \sim x^{2 / 3}$ and $v \sim-\Psi_{x}$. It clearly violates the assumptions from BLT that $v \sim R N^{-1 / 2}$, see Fig. 2 .

Although we will proceed with this approach we have to remark that this analytical model still is not sufficient for investigations on how the Kutta-Conditions emerges from viscous flows. Only if the flow velocity is at least continuous in all components we may be able to explain lift.

Analogously to Eq. (16) the wake is composed of two boundary layers (inner and outer wake) and therefore needs to be described in terms of two functions $H_{0}, H_{1}$ via:

$$
\begin{aligned}
3 H_{0}{ }^{\prime \prime \prime}+2 H_{0} H_{0}{ }^{\prime \prime}-{H_{0}}^{\prime 2} & =0, \\
3 H_{1}{ }^{\prime \prime \prime}+2 H_{0} H_{1}{ }^{\prime \prime}-5 H_{0}{ }^{\prime} H_{1}{ }^{\prime}+5 H_{0}{ }^{\prime \prime} H_{1} & =0 .
\end{aligned}
$$

Using $s=y / x^{1 / 3}$, it follows:

$$
\begin{aligned}
H_{0} & \sim \lambda_{0}^{2} s+\frac{\lambda_{0}^{4}}{33 !} s^{3}-\frac{2 \lambda_{0}^{6}}{95 !} s^{5}, \\
& H_{1} \sim \lambda_{1} \lambda_{0}\left(s--\frac{5}{18} \lambda_{0}^{2} s^{3}\right) \text { leading to } \\
u(x, 0) \sim x^{1 / 3}\left(\lambda_{0}^{2}+\lambda_{0} \lambda_{1} x\right) . &
\end{aligned}
$$

Numerical integration leads to [31] $\lambda_{0}=0.8789, \lambda_{1}=-0.1496$

Fig. 6 displays the combined flat plate and wake boundary layers together with the governing equations. At the trailing edge $v \sim x^{-1 / 3}$ as $x \rightarrow 0^{-}$.

\subsection{Triple Deck Theory}

The singularity close to $x=0$ can only be removed by introducing a three fold structure with an extension in $\mathrm{x} \sim R N^{-3 / 8}$, see Fig. 7 . 


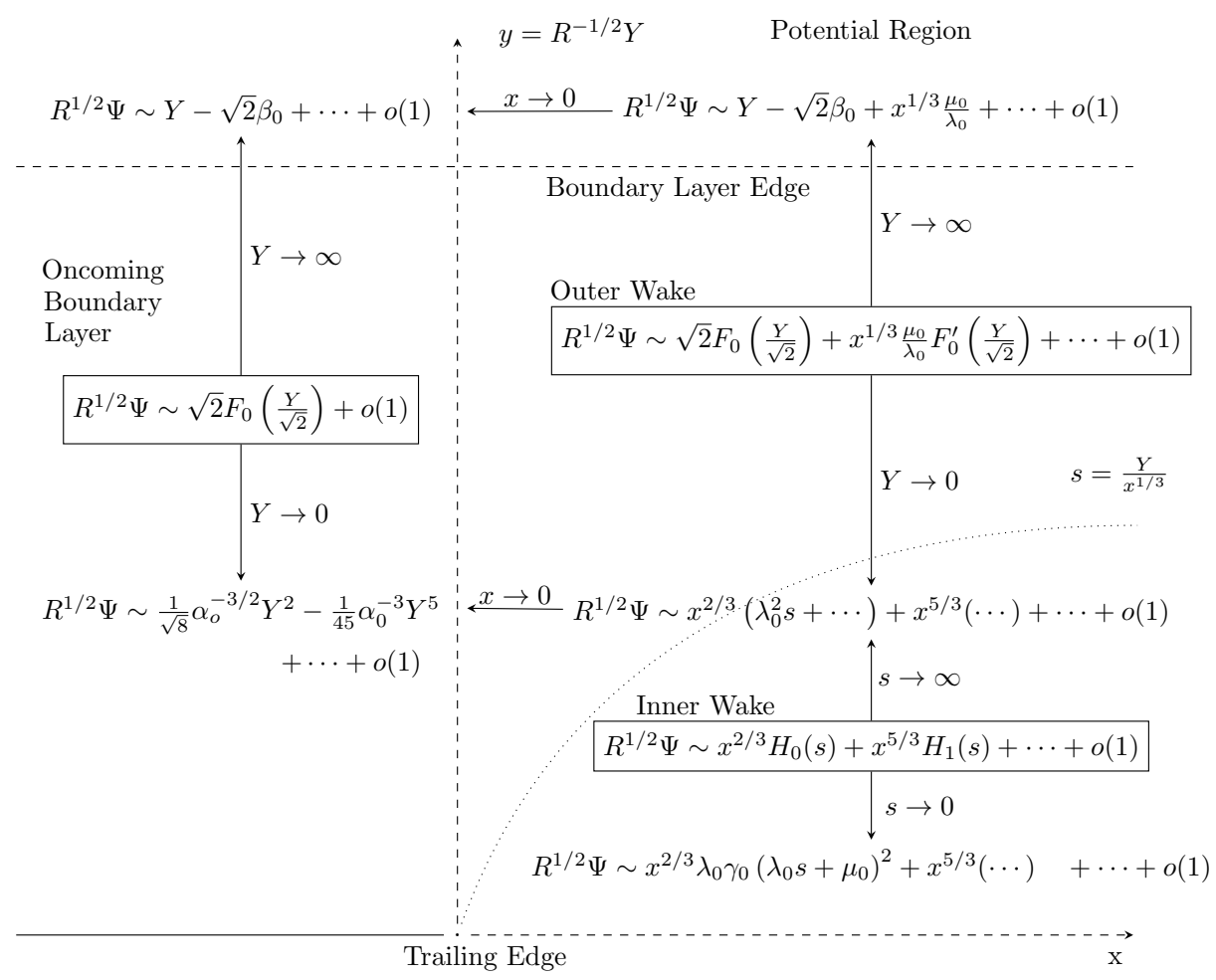

Figure 6. Goldstein's near wake structure. Adapted from [31]

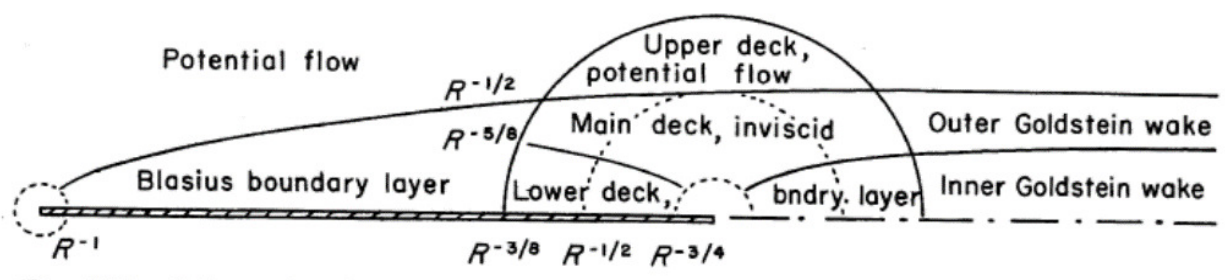

Fig. N.1. Schematic picture of matching regions for flow past finite flat plate at high Reynolds number; primary regions shown by solid lines and secondary regions by dashed lines.

Figure 7. Boundary Layer structure around the trailing edge of a flat plate, from [33] 


\subsection{Flat plate at zero incidence}

A considerable amount of work has to be done to remove the singularity mentioned above. First of all it is easy to show that the region close to the trailing edge - where BLT fails - scales according to $|x| \sim R e^{-3 / 8}$, see Sobey [31].

Improving the analytical solution? (in the sense of calculus) is now achieved by introducing the three-fold structure already mentioned above normal to the plate:

- Some kind of a viscous sub layer: the LOWER deck,

- A perturbation for the outer potential flow region in form of a displacement function $A_{1}(X)$ : the OUTER deck,

- And in between the MIDDLE (or MAIN) deck, sometimes called inviscid rotational disturbance layer.

A sketch of the structure is visualized in Fig. 7.

Triple Deck equations start with introducing appropriate scaled coordinates:

$$
\begin{array}{r}
\text { Main deck: } Y=R N^{1 / 2} y \\
\text { Inner deck: } Z=R N^{1 / 8} Y \\
\text { Outer deck: } W=R N^{-1 / 8} Y \text { and } \\
X=R N^{3 / 8} x .
\end{array}
$$

$A_{1}(X)$ is defined via $\psi_{1}^{m}(X, Y):=A_{1}(X) \cdot U_{0}(Y)$ and acts as a kind of a displacement function.

A characteristics set of equations can be derived [31]:

$$
\begin{array}{r}
u_{X}+v_{Z}=0 \text { (mass conservation) } \\
u u_{X}+v u_{Z}=-\frac{1}{\pi} \int_{-\infty}^{\infty} \frac{A_{1}^{\prime \prime}(\zeta)}{X-\zeta} d \zeta+u_{Z Z} ; \\
\text { together with asymptotics: } \\
u \rightarrow \frac{\alpha_{0}^{-3 / 2}}{\sqrt{2}}\left[Z+A_{1}(X)\right] \text { as } Z \rightarrow \infty, \\
A_{1}(X) \rightarrow 0 \text { as } X \rightarrow-\infty, \\
A_{1}(X) \rightarrow \frac{\mu_{0}}{\lambda_{0}} X^{1 / 3} \text { as } X \rightarrow \infty .
\end{array}
$$

In [31] FORTRAN routines for solving this non-liner set of integro-differential equations are given. Some sample results are presented in Fig. 8 and Fig. 9. As can be seen from the plots, all functions now are continuous at $x=0$ (trailing edge) but still are not $\in \mathcal{C}^{1}$ (continuous slope). For reasons of comparison we added results from CFD in Fig. 9.

Pressure is shown in Fig 9 together with two asymptotics $p \sim$

$$
\begin{gathered}
-\frac{2}{3 \sqrt{3}} \frac{\mu_{0}}{\lambda_{0}} R N^{-1 / 2}|x|^{-2 / 3} x<0, \\
\frac{1}{3 \sqrt{3}} \frac{\mu_{0}}{\lambda_{0}} R N^{-1 / 2} x^{-2 / 3} x>0 .
\end{gathered}
$$

Singularity $\mathrm{v} \rightarrow \infty$ at the trailing edge (see Eq. 27) disappears, because [34]

$$
v \sim-A_{1}{ }^{\prime}(x),
$$

is finite. Unfortunately, streamlines close to the TE are not $\in \mathcal{C}^{1}$ (space of functions which are once continuous differentiable). Shifting this dis-continuity to even higher derivatives demands introduction of even more structure in form of more sub-layers [31,35].

Nevertheless, one of the greatest success of TDT is an impressive improvement of prediction of finite length flat flat flate-plate? drag coefficient. We will present these 
Triple Deck Displacement Function A1(X)

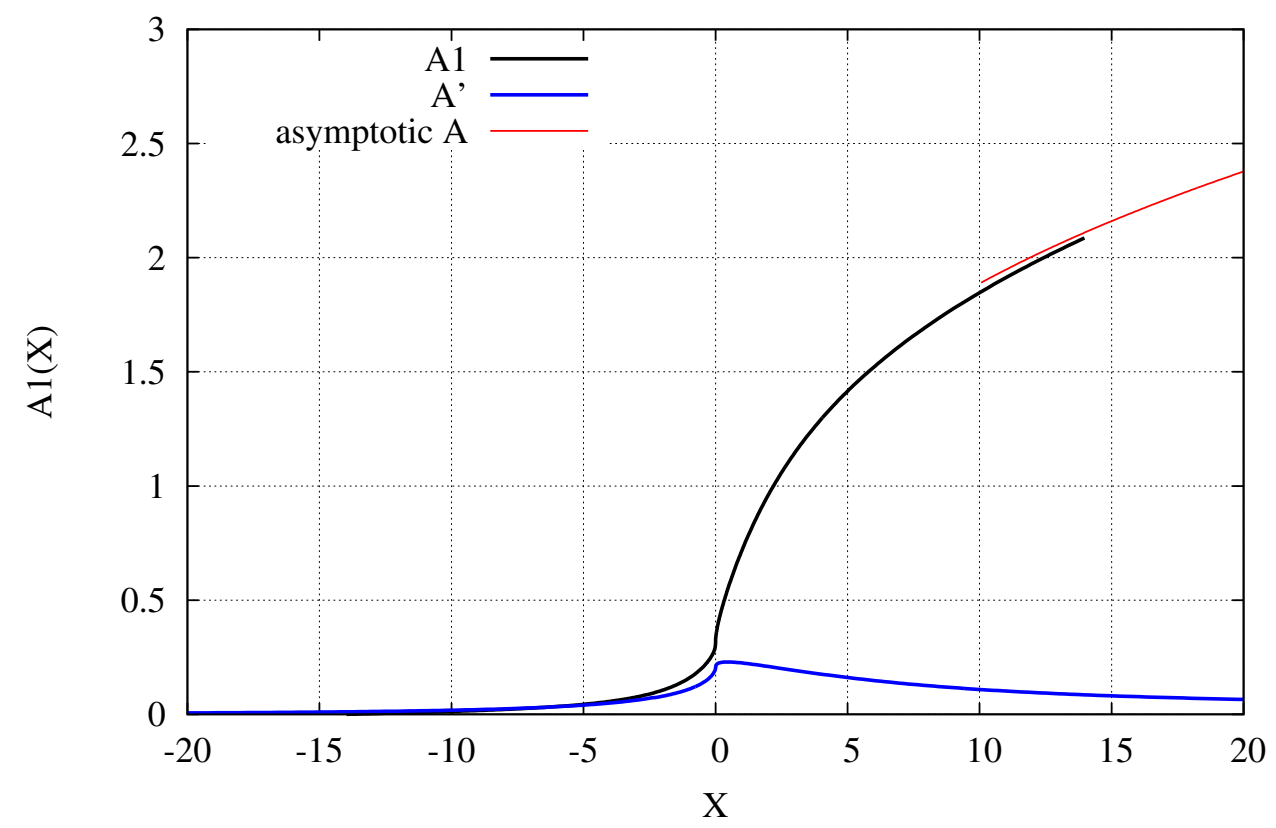

Figure 8. Triple Deck displacement Function $A_{1}$ calculated with help of a FORTRAN code published by [31]. To scale $A_{1}$ in SI units it has to be multiplied by $R N^{-3 / 8}$. If $\mathrm{RN}=10^{6}$ the scale then is $5.6 \cdot 10^{-3}$. $A_{1}$ must obey the asymptotic limit $\frac{\mu_{0}}{\lambda_{0}} X^{1 / 3}$ as $X \rightarrow \infty$ (red line). In addition $A_{1}{ }^{\prime}$ is included which is $\in \mathcal{C}^{0}$ (continuous) but $\notin \mathcal{C}^{1}$.

Triple Deck Presure

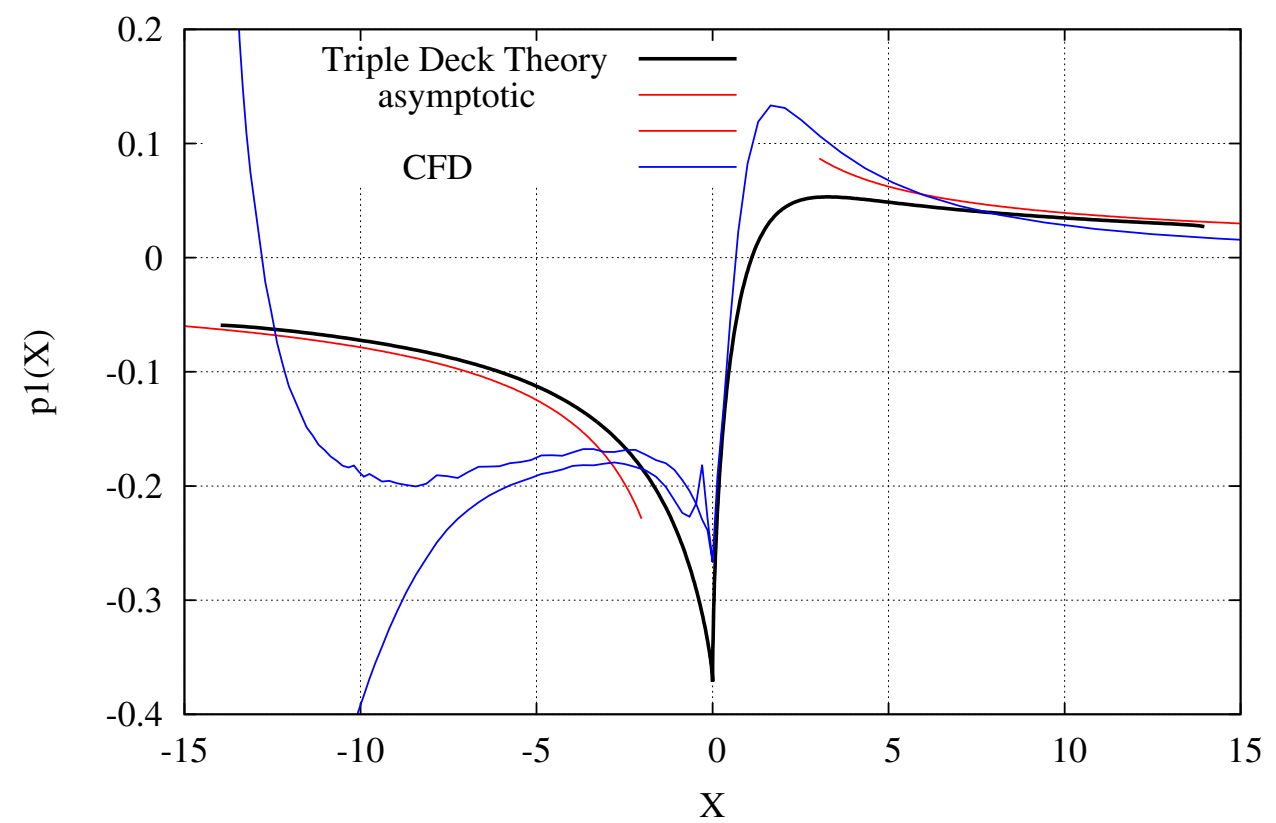

Figure 9. Pressure around trailing edge from triple-deck-theory [31] (black), together with asymptotic values from Blasius and Goldstein (red) and CFD for a thin airfoil (blue) see section 4 . The two blue line upstream to the trailing edge correspond to the upper and lower side of the airfoil. Note that the pressure around the airfoil calculated by CFD is not scaled. 
Triple Deck Presure

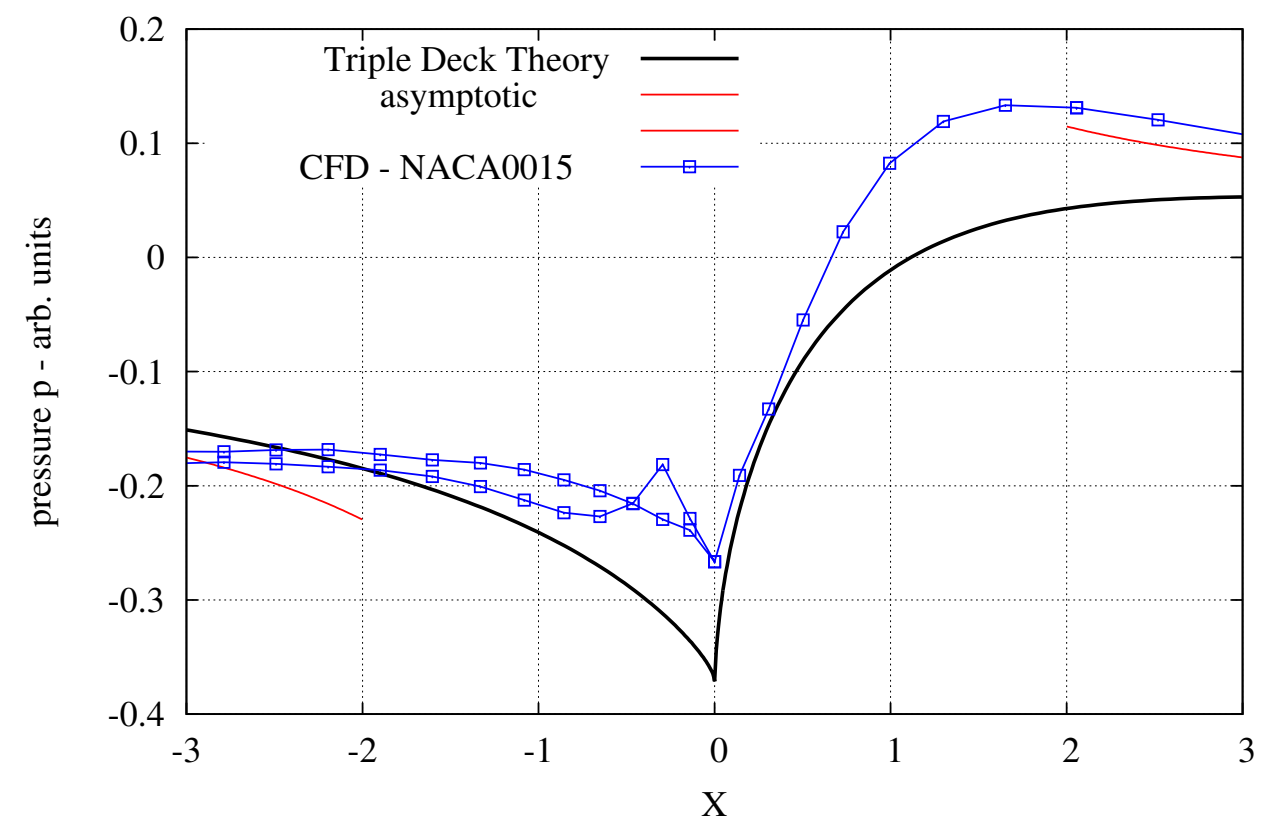

Figure 10. Same as Fig. 9, but enlarged. The apparently out-liner at $X \approx-0.29$ might be due to inaccurate geometric modelling of the profile.

findings in more detail in section 4.

\subsection{Flat Plate at an Incidence and Embedding of the Kutta Condition}

The final step now is to apply the findings from section 3.5 to a flat plate of finite length and non-zero angle-of-attack which severs as a simple model of a 2D airfoil. This has been done already 50 years ago by Brown and Stewardson [5]. A summary of the historic development is presented in [35].

Apart from the discontinuity of the viscous boundary layer condition at the edge - a zero tangential velocity on the plate $\left(x \rightarrow 0^{-}\right)$faces a zero pressure discontinuity on the wake center-line $\left(x \rightarrow=0^{+}\right)$- the phenomenon of separation comes into play.

The following set of steps are necessary to derive the Kutta-Joukovsky-condition and a viscous correction to the lift coefficient:

Introduce triple deck length scale:

$\epsilon=R N^{-1 / 8}=\left(U_{\infty} \ell / v\right)^{-1 / 8}$

Length scale Kutta conditon violation :

$-x^{\star} / \ell=\epsilon^{7} \alpha^{1} a_{1}^{2} \lambda^{-1 / 4}$

Angle-of-attack: $\alpha \sim \mathcal{O}\left(\epsilon^{1 / 2}\right)<5^{\circ}$

Blasius wall shear stress: $\lambda=0.3321$.

This leads to:

$$
\begin{aligned}
& \text { Lift: } c_{L}=2 \pi \alpha\left(1-\frac{2 B}{\ell}\right) \\
& \text { Circulation term: } B=\epsilon^{3} \ell \lambda^{-5 / 4} \cdot a_{1}
\end{aligned}
$$




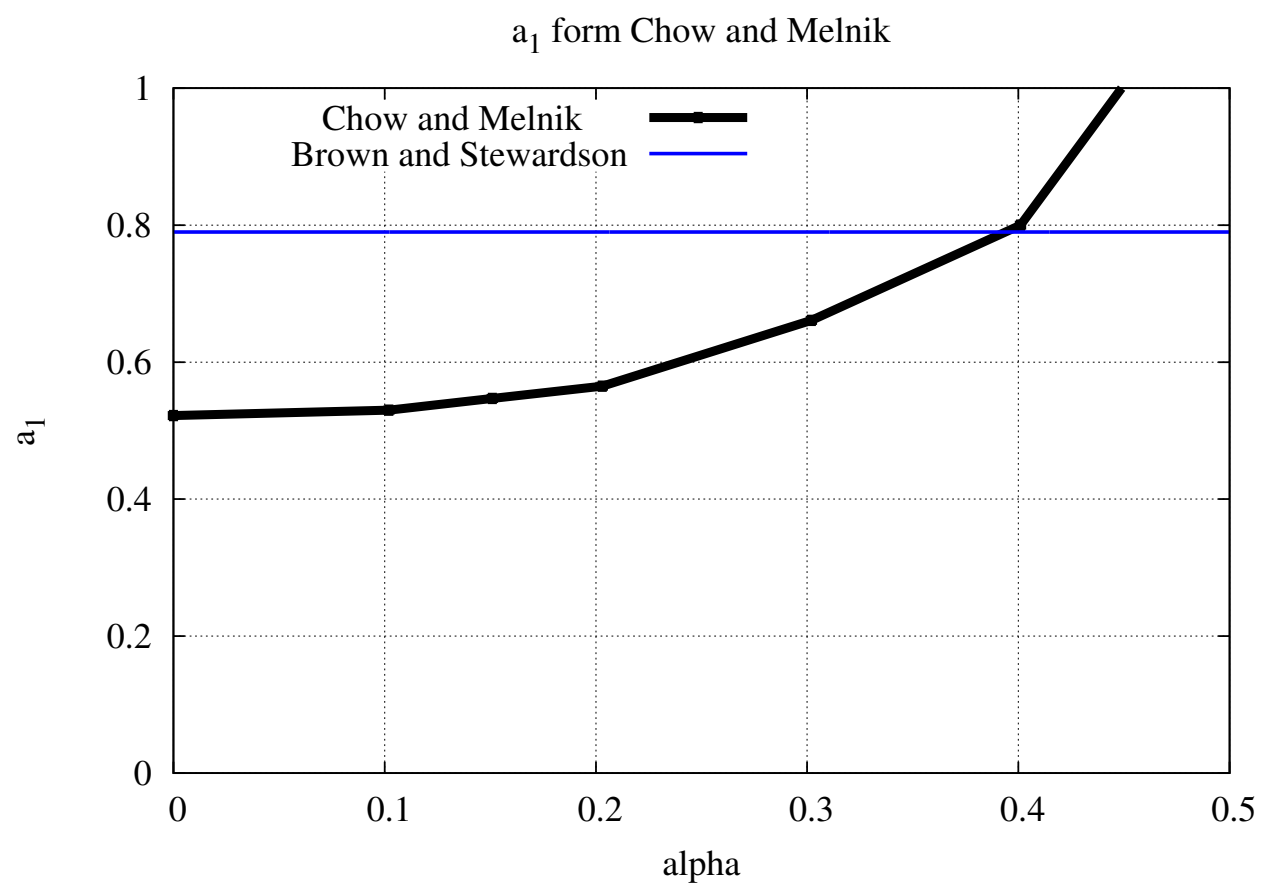

Figure 11. Variation of the parameter $a_{1}$ with (reduced) angle-of-attack from [36]. As $a_{1}$ approches 0.47 this number diverges, indicating flow separation.

At the time of [5] no computer codes for solving the set of equations Eq. 37 to Eq. 41 was available. This occurred only in 1976 with the paper [36]. Instead some kind of linearisation in the form of a Hilbert transform was used. The constant $a_{1}$ from 52 was analytically estimated to

$$
a_{1}=2^{-1 / 2} \gamma^{-3 / 4} \cos \left(\frac{\pi}{8}\right)=0.79 \quad \text { with } \gamma=3^{2 / 3} / \Gamma(1 / 3)=0.7764
$$

Later Chow and Melnik [36] improved the value for $a_{1}$ from a constant value (0.79) by Brown/Stewardson [5] to one dependent on the AOA, see Fig. 11. Thereby, separation is predicted for AOAs larger than $\alpha_{S}>0.47$, which - in degrees - corresponds to rather small values of $3.8^{\circ}$ for $R N=10^{5}$ Quoting Crighton [37] this approach provides detailed analytical and computational understanding (emphasis by the present author) as it gives a smooth transition from the flat plate boundary layer $\sim R N^{-1 / 2}$ to Golstein's wake $\sim R N^{-1 / 2} \cdot x^{1 / 3}$ already visualized in Fig. 9.

\subsection{Turbulent boundary layers}

The restriction of laminar boundary layers certainly forbids applications for $R N>$ $5 \cdot 10^{5}$. Therefore it is tempting to try to apply methods from TDT to turbulent boundary layers. This has been first attempted by Melnik and Chow [38] and is further discussed in $[39,40]$. As a result Cousteix and Mass [40] conclude that - because no overlapping layer (the famous logarithmic law of the wall) exist, a chosen turbulence model has to be restricted to those which leads us to the desired result.

\section{Comparison with CFD}

We have prepared computational meshes for two cases:

- A flat plate of unit length

- A simple 2D aerodynamic profile (NACA0015) 


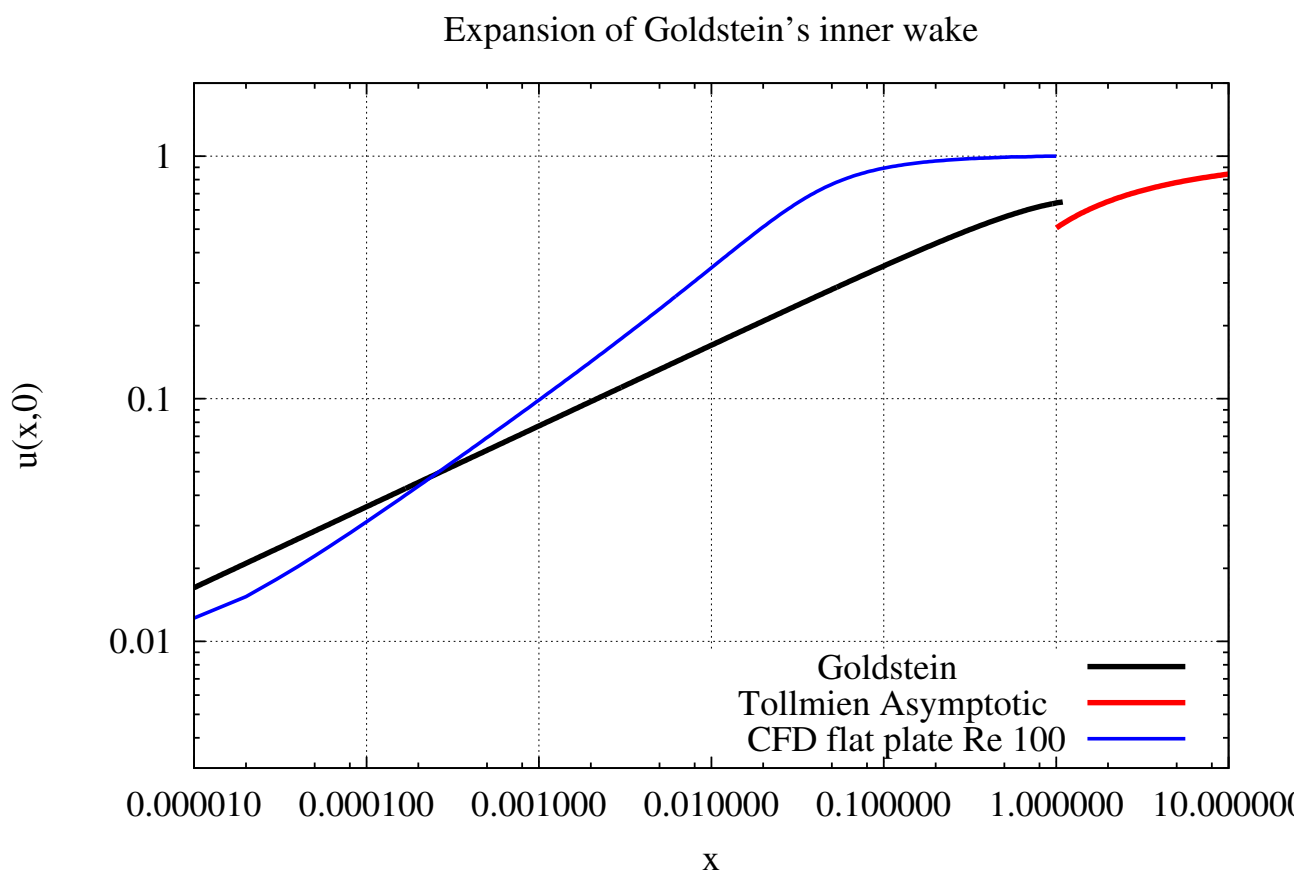

Figure 12. Center line velocity $u(x, y=0)$ from Eq. (32) compared to a CFD model calculation. Note the doulbe-logarithmic scaling of the axes and that the abscissa (scales according to TDT) covers 7 orders of magnitude. CFD profile reaches much earlier the asymptotic value, indicating a two small computational area.

\section{As a solver we use ANSYS-FLUENT V18}

\subsection{Finite Length Flat Plate}

Fig. 12 shows the development of the wake at $y=0$ for $x \geq 0$ from TST and full CFD. The deviation may be caused by a too small calculational area (10 chords only).

To have a more quantitative comparison of TDT we compared highly sensitive drag data either calculated from the above mentioned CFD-model or from equations derived by TDT by different variants:

$$
\begin{aligned}
& \text { Imai: } \quad c_{D} \sim \frac{1.33}{(R x)^{1 / 2}}+\frac{2.32}{R x}-\frac{2.20}{(R x)^{3 / 2}} \log \sqrt{R x}+\mathcal{O}\left(R^{-3 / 2}\right) \text { and } \\
& \text { Dean: } \quad c_{D} \sim \frac{1.33}{\sqrt{R x}}+\frac{2.32}{R x}+\mathcal{O}\left(R^{-3 / 2}\right) .
\end{aligned}
$$

as shown in table 1. Unfortunately, the contribution of the next-to-BL term $\sim R N^{-7 / 8}, c_{2}$

Table 1: Comparison of calculated flat plate drag data for various RN compared to CFD

\begin{tabular}{|l|l|l|l|l|} 
RN & $c_{D}$ & $c_{2}$ & $c_{D}$ Imai & $c_{D}$ Dean \\
\hline $1.23 \cdot 10^{5}$ & $3.9 \cdot 10^{-3}$ & 3.04 & $3.81 \cdot 10^{-3}$ & $3.82 \cdot 10^{-3}$ \\
\hline $1.0 \cdot 10^{4}$ & $1.43 \cdot 10^{-2}$ & 3.20 & $1.34 \cdot 10^{-2}$ & $1.35 \cdot 10^{-2}$ \\
\hline $1.0 \cdot 10^{2}$ & $1.92 \cdot 10^{-1}$ & 3.31 & $1.14 \cdot 10^{-1}$ & $1.56 \cdot 10^{-1}$ \\
\hline
\end{tabular}

can only be compared at a $10 \%$ level. Even worse several hundreds of thousands of CFDiterations have to be performed to be able to use a Richardson-type of extrapolation for the drag-coefficient. The meaning of this huge computational effort is clear: accurate CFD calculations to compare with accurate analytical theories demand heavy resources: 
Lift coefficient NACA0009

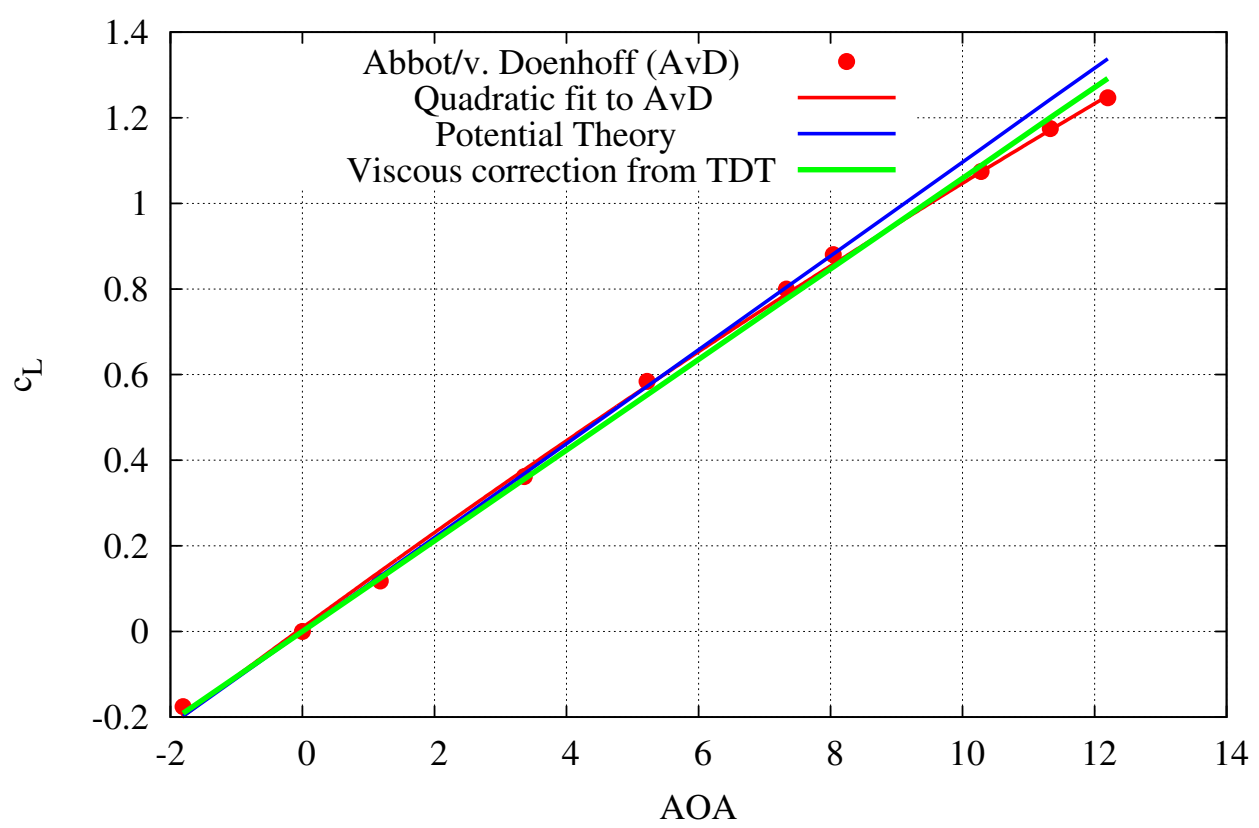

Figure 13. Lift coefficient of NACA0009 as function of angle of attack at RN=6 $10^{6}$ together with potential theoretic prediction and viscous correction from TDT.

An example for $\mathrm{RN}=100$ : After $N_{\text {iter }}=250 \mathrm{k}$ iterations drag force was 0.1307 , but an extrapolation to $N_{\text {iter }} \rightarrow \infty$ lowers this value by about $20 \%$ to 0.1174 .

\subsection{Thin Symmetric NACA Profile}

As a last example to scrutinize the validity and accuracy of TDT we compare viscous correction from TDT with measuremnts for a 9\%-thin NACA airfoil, see Fig. 13. The measurements show some scatter but has been fitted to a simple three-parameter parabolic shape. As can be seen TDT (Eqs.57 to 59) describe the lowering of the lift-coefficient with some accuracy. It has to be noted that a somewhat thicker profile (NACA0012) has been investigated by Cebeci and Cousteix [? ].

\section{Summary and Conclusions}

Within the framework of matched asymptotic expansion, boundary layer theory can be extended to match Goldstein's wake to Blasius' flat plate boundary layer. With the use of $\epsilon=R N^{-1 / 8}$ as an expansion parameter Brown and Stewardson [5] were the first who presented a physical picture of the Kutta condition together with a quantitative viscous correction (in terms of a parameter B) for the slope of the lift coefficient:

$$
\begin{aligned}
\frac{c_{L}}{2 \pi \alpha} & =1-B, \\
B & =a_{1} \cdot \lambda^{-5 / 4} \epsilon^{3},
\end{aligned}
$$

with $0.508 \leq a_{1} \leq 1$ for $0 \leq \alpha \leq \alpha_{S}(: / /$ www.overleaf.com/project/609a5270c5eac87a761a1b

Therefore, the wake with its continuous pressure in y-direction enforces an equal continuous pressure for $y=0$ across the trailing edge in $x$-direction and induces fixed (and finite) velocities, circulation and lift [35].

We now present the resulting physical picture emerging from the circulation approach and higher order boundary layer theory (triple-deck theory):

- A Kutta-Joukovsky condition fixes circulation and thereby lift.

- Stated mathematically, it demands a finite velocity at the trailing edge. 
- The simplest model of a lifting surface consists of a flat plate of finite length and a non-zero angle of attack.

- Matching Blasius' boundary layer with Goldstein's wake needs an intermediate triplestructured layer of length of $R N^{-5 / 8}$ to interpolate between both different boundary conditions.

- Worked out, a set of equations results which predicts finite velocities and pressure around the trailing edge but a non-continuous pressure gradient.

- A viscous correction to the potential-theoretic lift coefficient slope of $2 \cdot \pi$ can be derived and compared to experimental data.

- Extension to turbulent boundary layers is possible but relies on the closure models.

\section{Acknowledgements}

Stimulating discussions with I.J. Sobey, University of Oxford, Oxford, UK and S. Braun, The Technical University of Vienna, Vienna, Austria are gratefully acknowledged. Gijs van Kuik, Delft Technical University, Delft, The Netherlands helped in reviewing an early version of this paper. Brandon Lobo, Kiel University of Applied Sciences prepared Fig. 6 and helped to improve the text.

\section{Abbreviations}

Table 2: The following abbreviations have been used in this manuscript

$\begin{array}{ll}\text { AOA } & \text { Angle of attack } \alpha \\ \text { BLT } & \text { Boundary layer theory } \\ \text { CFD } & \text { Computational Fluid Dynamics } \\ \text { M } & \text { Million } \\ \text { RANS } & \text { Reynolds Averaged Navier Stokes (Equations) } \\ \text { RN } & \text { Reynolds Number } \\ \text { TAT } & \text { Thin Airfoil Theory } \\ \text { TDT } & \text { Triple Deck Theory } \\ \text { TE } & \text { Trailing Edge } \\ c_{L} & \text { Lift coefficient } \\ c_{D} & \text { Drag coefficient }\end{array}$

Conceptualization, methodology, software, validation, formal analysis, investigation, resources, data curation, writing_-original draft preparation, writing—-review and editing, visualization, supervision and project administration by the author. The author has also read and agreed to the published version of the manuscript.

Funding: This research received no external funding

Conflicts of Interest: The author declares no conflict of interest.

\section{References}

1. Regis, E. The Enigma of Aerodynamid Lift. Sci.Am. 2020, 322(2), 44 - 51.

2. McLean, D. Understanding Aerodynamics Arguing from the Real Physics; Wiley, 2013.

3. McLean, D. Aerodynamic lift, Part 1: The science. Phys. Teach. 2018, 56, 516-520.

4. McLean, D. Aerodynamic lift, Part 2: Comprehensive Physical Explanation. Phys. Teach. 2018, $56,521-524$.

5. Brown, S.; Stewardson, K. Trailing-edge stall. J. Fluid Mech 1970, 42,3, $561-584$.

6. van Kuik, G. The Fluid Dyanmic Basis for Actuator Disc and Rotor Theories; IOS Press BV, Amsterdam, The Netherlands, 2018.

7. Landau, L.; Lifshitz, E. Fluid Mechanics, 2nd Edition; Pergamon Press, 1987.

8. Bloor, D. The enigma of the aerofoil; University of Chicago Press, 2011.

9. Batchelor, G. An Introduction to Fluid Dynamics; Cambridge University Press, 1967. 
10. L. Howarth. The Theoretical Determination of the Lift Coefficient for a Thin Elliptic Cylinder. Proc Roy Soc A 1935, 149, 558-586. doi:10.1098/rspa.1935.0081.

11. M. Drela. XFOIL: An Analysis and Design System for Low Reynolds Number Analysis. Springer Notes in Engineering 1989, 54, 1267-1289. doi:10.10107/978-3-642-84010-4-1.

12. W R Sears. Some Recent Developments in Airfoil Theory. J Aeor Sci 1956, 23, 5, 490-499. doi: $10.2514 / 8.3588$.

13. J Y Zhu and T S Liu and L Liu and S F Zhou and J Z Wu. Causal mechanism in airfoil-circulation formation. Phys Fluids 2015, 27. doi:10.1063/1.4937348.

14. Jones, T. Wing Theory; Princeton University Press, 1990.

15. Freudenreich, K.; Kaiser, K.; Schaffarczyk, A.; Winkler, H.; Stahl, B. Reynnolds Number and Roughness Effects of Thick Airfoils for Wind Turbines. Wind Engineering 2004, 28.

16. Yates, J.E. A Unified Viscous Theory of Lift and Drag of 2-D Thin Airfoils and 3-D Thin Wings. Technical Report CR-4414, NASA, 1991.

17. L W Bryant and D H Williams. An Investigation of th Flow of Air Around an Aërofoil of Infinite Span. Phil Tran Roy Soc A 1926, 225, 626-635. doi:10.1098/rsta.1926.0005.

18. S F Shen and P Crimi. The theory for an oscillating thin airfoil as derived from the Oseen equations. J. Fluid Mech. 1965, 23, 585-609. doi:10.1017/S0022112065001568.

19. Schlichting(deceased), H.; Gersten, K. Boundary Layer Theory, 9th Ed.; Springer, 2017.

20. Abbott, I.; von Doenhoff, A. Theory of wing sections: Including a summary of airfoil data; Dover, 1959.

21. J E Yates. Viscous Thin Airfoil Theory and the Kutta Condition. AIAA 16th Aerospace Science Meeting, 1978, pp. 78-152.

22. Yates, J.E. Viscous Thin Airfoil Theory. Technical Report 413, Aeronautical Research Associates of Princeton Inc., 1980.

23. S. Schmitz. Finite Domain Viscous Correction to the Kutta-Joukowski Theorem in Incompressible Flow. AIAA J 2014, 52, 9, 2079-2083. doi:10.2514/1.J053114.

24. S. Schmitz and J. G. Coder. Inviscid Circulatory-Pressure Field Derived from the Incompressible Navier-Stokes Equations. AIAA J 2015, 53, 1, 33-41. doi:10.2514/1.J053140.

25. Schmitz, S. Aerodynamics of Wind Turbines; Wiley, 2020.

26. Prandtl, L. Über Füssigkeitsbewegung bei sehr kleiner Reibung. Verh. III. Math.-Kongr., Heidelberg; Druck und Verlag, B.G. Teubner, L.G., Ed., 1905, pp. $484-491$.

27. Blasius, H. Grenzschichten in Flüssigkeiten mit kleiner Reibung. Z. Math. Phys. 1908, 56,1, 1-37.

28. Weyl, H. On the Differential Equations of the simplest Boundary-Layer Problems. Ann Math, 2, $381-407$.

29. Boyd, J. The Blasius Function in the Complex Plane. Exp.Math. 1999, 8/4, 381 - 394.

30. Boyd, J. The Blasius Function: Computations Before Computers, the Value of Tricks, Undergraduate Projects, and Open Research Problems. SIAM reviews 2008, 50/4, 791-804.

31. Sobey, I. Introduction to Interactive Boundary Layer Theory; Oxford University Press, Oxford, UK, 2000.

32. Goldstein, S. Concerning some Solutions of the Boundary Layer Equations in Hydrodynamics. Proc. Camb. Phil. Soc. 1930, XXVI,I.

33. Van Dyke, M. Pertubation Methods in Fluid Mechanics; The Parabolic Press, Stanford, CA, USA, 1975.

34. Braun, S. Recent developments in the asymptotic theory of separated flows. Technical report, Leverhulme lectures - lecture notes, Manchester, UK.

35. Sychev, V.; Ruban, A.; Sychev, V.; Korolev, G. Asymptotic Theory of Separated Flows; Cambridge University Press, Cambridge, UK, 2008.

36. Chow, R. and Melnik, R.E. Numerical Solution of the Triple-Deck Equations for Laminar TrailingEdge Stall. Proc. 5th Internationla Confernce on Numerical Methods in Fluid Dynamics, 1976, pp. 135-144.

37. Crighton, D. The Kutta Condition in Unsteady Flow. Ann. Rev. Fluid Mech. 1985, 17, $411-445$.

38. Melnik, R.E and Chow, R.. Asymptotoc Theory of Two-Dimensional Trailing-Edge Flow. NASA SP-347, NASA, 1975.

39. Cousteix, J.; Mauss, J. Rational Basis of the Interactive Boundary Layer Theory. One Hundred Years of Boundary Layer Research; Meier, G.; Sreenivasan, K., Eds., 2004, pp. 29-38.

40. Cousteix, J.; Mauss, J. Asymptotic Analysis and Boundary Layers; Springer-Velag Berlin Heidelberg, 2007. 